\title{
A qualitative investigation of the 'reality' of living with lifestyle management of type 2 diabetes mellitus: the patient's perspective
}

\author{
Lucie Nield and Helen Moore \\ University of Teesside, Middlesbrough, UK
}

A number of interviews were planned with the aim of providing an insight into what dietary advice, treatment and recommendations were given to patients to treat their type 2 diabetes and to discover how well this advice was understood, the reality of living with diabetes and the actual compliance with dietary and lifestyle advice.

For this pilot study five in-depth semi-structured qualitative interviews were conducted in June and July 2007 in the Teesside region, UK. The interviews were based on a topic guide developed by the researchers, to guide the flow of conversation. Interviews were carried out in the participants' own homes.

The participants were of varying age, gender and time since diagnosis (of type 2 diabetes mellitus) and were recruited by email, letters and word-of-mouth facilitated by colleagues of the University of Teesside. A semi-structured qualitative interview was used to get an indepth description of the participants' experiences of living with diabetes and changes in their dietary and lifestyle habits. The interviews were transcribed and coded with thematic analysis, which was carried out by two reviewers to reduce bias. Themes were then extracted, compared, analysed, interpreted and reported.

Six main themes were identified: emotions and feelings on diagnosis; short- and long-term compliance (including medication and prescribed treatments, self-care, dietary and lifestyle advice and guilt); food choices and the diabetic diet (including giving up treats); blood glucose monitoring; clinical advice and group sessions and rapports with healthcare providers (including honesty).

Patients appear to fully understand the advice that they have been given, and know what they should be doing, but still fail to follow the advice. Focus should be based, therefore, not on the advice provided, but on the methods used to supply this advice, and the refresher and follow-up sessions thereafter. The present research demonstrates the lack of compliance that this sample population have to their dietary management, blood glucose monitoring and self-care, which is probably an insight into what is happening nationally, and internationally, within the diabetic population. There is a demand for follow-up sessions, not just as individual check-ups, but through group sessions, which were found to be most beneficial.

The small sample size and limited demographics of the sample group is a weakness in the present study, but evidence of similar themes has been found in other research ${ }^{(1)}$, and it is believed that these themes are key to this demographic. It is believed that these findings could be used as a basis for a larger national qualitative research project to investigate the reality of living with diabetes, weaknesses in the current treatment and service options available, and to develop new treatment strategies. There is still much research that needs to be carried out, with the aim of improving the service for patients with diabetes, improving their compliance and self-care and, most importantly, improving their health and delay or prevention of further diabetic complications.

1. Dietrich UC (1996) Patient Educ Couns 29, 13-23. 